Fig. 2, curve c). This has been interpreted as evidence for a complex formation of the reactants. The minimum results because the complex lifetime which determines the reaction probability, is an inverse function of the relative velocity. At higher energies, the increase of the rate-constant is generally explained by the opening of endoergic channels.

Another class of ion-molecule reactions where the simple Langevin model cannot be applied involves polar molecules. At sub-thermal energies, a dramatic increase of the rate-constant is observed reaching values much higher than $k_{\mathrm{L}}$. This has been explained by taking into account the fact that for polar molecule reactions, the collinear collision geometry is the most favourable. Rotational excitation will prevent this configuration. Thus an increase of rotational temperature will lead to a reduction of the reaction rate-constant. The finding has of course a strong impact on models explaining molecular synthesis in interstellar clouds.

\section{Vibrational Effects}

In the case of molecular ions it has been observed that drift experiments carried out with different buffer gases yield often different values of the reactive rate-constant. This fact has been related to the vibrational excitation (and quenching) of molecular ions by collision with the buffer gas (see W. Lindinger in Ref. [1]). The effect of vibrational energy on reactivity can been demonstrated in a direct and elegant way by coincidence photo-ionization techniques. In these experiments, detection of threshold electrons in coincidence with product ions enables an accurate determination of the quantum state of the reactant ions to be made [8].

Advances in the study of vibrational effects have made possible the development of the so-called "ion-monitor" technique [9]. This method has found wide application in drift experiments to analyze the vibrational energy distribution of ions. Recently in Orsay it has been applied also to ICR spectrometry for studying the radiative decay of vibrationally excited ions (see G. Mauclaire et al. in Ref. [1]). The method consists of using specific ion-neutral reactions which are strongly dependent on the vibrational state of the reactant ion. For these reactions the number of product ions is a measure of the number of vibrationally excited ions. As an example the fraction of molecular nitrogen ions with vibrational number $v \geq 1$ can be measured by the charge- transfer reaction:

$$
\mathrm{N}_{2}^{+}+\mathrm{Ar} \rightarrow \mathrm{Ar}^{+}+\mathrm{N}_{2}
$$

For the ground state $\mathrm{N}_{2}^{+}(X, v=0)$ ions this reaction is endoergic by about $0.18 \mathrm{eV}$ and does not proceed at a significant rate. A dramatic increase of the reaction rate-constant is observed when $\mathrm{N}_{2}^{+}$ions are vibrationally excited: the $\mathrm{Ar}^{+}$signal can then be used to estimate the fraction of $\mathrm{N}_{2}^{+}(X, v \geq 1)$.

Chemical diagnostics of ion vibrational populations has made possible in the last few years the systematic study of vibrational quenching (and excitation) of diatomic ions in collision with neutrals. The large value of quenching rate-constants and its decrease with the increase of collision energy have been interpreted as due to the fact that collisions are driven by the long-range attractive tail of the potential and that transfer of vibrational energy occurs through the formation of a transient complex followed by vibrational pre-dissociation (see Fig. 3). This is an extension of the bound-free vibrational predissociation of Van der Waals molecules to the free-free binary collision limit. In cases where a large attraction does not exist, vibrational relaxation is inefficient and its probability increases with relative collision energy. In this case the energy transfer arises from the short range repulsive forces. The criterion for whether the attractive or the repulsive potential will dominate quenching must relate to the ratio between the well depth $\varepsilon$ and the relative collision energy $K E_{\mathrm{cm}}$. If $K E_{\mathrm{cm}} \ll \varepsilon$, attractive forces are expected to dominate the quenching process. On the contrary if $K E_{\mathrm{cm}} \gg \varepsilon$, vibrational relaxation will be driven by the repulsive potential. In experiments where $K E_{\mathrm{cm}}$ can be varied, one should observe a minimum in the quenching rate-constant as a function of $K E_{\mathrm{cm}}$. So far such a minima have been reported for $\mathrm{O}_{2}^{+}$in collision with $\mathrm{Kr}[10]$ and $\mathrm{NO}^{+}$ in collision with $\mathrm{CH}_{4}$. Extensive studies in this field are currently in progress in several European laboratories (Orsay, Innsbruck, Aberystwyth and Trento).

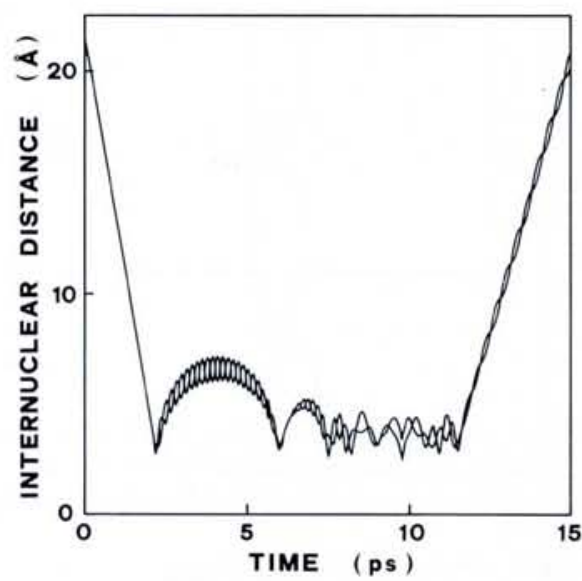

Fig. 3-Example of a trajectory leading to vibrational relaxation of $\mathrm{O}_{2}^{+}$in collision with $\mathrm{Kr}$ through the formation of a transient complex. Internuclear $\mathrm{Kr}-\mathrm{O}$ distances are shown as a function of time. The intersection between the two lines is due to $\mathrm{O}_{2}^{+}$ rotation. Collision energy is $0.04 \mathrm{eV}$.

\section{REFERENCES}

[1] A special issue dedicated to Prof. E.E. Ferguson; Eds. N.G. Adams and D. Smith, Int. J. Mass Spectrom. Ion Processes $\mathbf{8 0}$ (1987) 81.

[2] Atomic and Molecular Beam Methods, Ed. G. Scoles; Ass. Eds. D. Bassi, U. Buck and D. Laine, Vol. 1 (Oxford University Press, New York) 1988.

[3] Gaseous Ion Chemistry and Mass Spectrometry, Ed. J.H. Futrell (Wiley, New York) 1986.

[4] Tosi P., Fontana G., Longano S. and Bassi D., Int. J. Mass Spectr. Ion Processes (1989) (in press).

[5] Swarms of lons and Electrons in Gases, Eds. W. Lindinger, T.D. Märk and F. Howorka (Springer, Wien) 1984.

[6] Clary D.C., Smith D. and Adams N.G., Chem. Phys. Letters 119 (1985) 320.

[7] Bohringer $\mathrm{H}$. and Arnold F., Int. J. Mass Spectrom. Ion Phys. 49 (1983) 61.

[8] Govers T.R., Guyon P.M., Baer T., Cole K., Frohlich H. and Lavollee M., Chem. Phys. 87 (1984) 373.

[9] Ferguson E.E., J. Phys. Chem. 90 (1986) 731.

[10] Tosi P., Ronchetti M. and Laganá A., J. Chem. Phys. 88 (1988) 4814.

\title{
Physical Society of Japan
}

Members of the EPS are welcome to present their original papers at the 1990 Annual Meeting of the Physical Society of Japan to be held at Osaka University from 30 March - 2 April 1990. Application deadline for the presentation is 4 December 1989. EPS Members are entitled to the same privileges as members of the PSJ which is a Collaborating Society of EPS. Details may be obtained from:
The Physical Society of Japan Room 211, Kikai-Shinko Building 3-5-8 Shiba-Koen, Minato-Ku Tokyo 105, Japan

EPS Members may also subscribe to the Journal of the Phys. Soc. Jap. at the same rates as PSJ members. Subscription rates for vol. 59 (1990) are:

* 14000 surface mail ¥39000 airmail.

Orders should be addressed to the PSJ at the address shown above. 